\title{
Treino em automonitoração e comportamentos de prevenção de diabetes tipo $2^{1}$
}

\author{
Self-monitoring training and diabetes \\ type 2 prevention behavior
}

\author{
Mariene da Silva CASSEB ${ }^{2}$ \\ Eleonora Arnaud Pereira FERREIRA ${ }^{3}$
}

\begin{abstract}
Resumo
Este estudo investigou os efeitos de um treino em automonitoração na instalação e ampliação de repertórios comportamentais considerados como preventivos para a ocorrência de diabetes. Descreve-se um caso clínico realizado com uma mulher adulta com os seguintes fatores de risco: alimentação inadequada, sobrepeso, sedentarismo e histórico familiar de cardiopatia e diabetes. Foram realizadas visitas domiciliares para investigação de características sociodemográficas, levantamento de linhas de base do comportamento alimentar e de atividade física, treino em automonitoração e entrevista final. Os resultados apontam mudanças comportamentais referentes à instalação e à ampliação de hábitos alimentares mais saudáveis e prática regular de atividade física. A discussão do caso abrange aspectos que indicam a aquisição de comportamentos preventivos, relacionando-os com a literatura utilizada.
\end{abstract}

Unitermos: Diabetes. Redução de fatores de risco. Treino em automonitoração.

\begin{abstract}
This study investigated the effects of self-monitoring training for the implementation and expansion of behavioral repertoires considered as preventive in the occurrence of diabetes. One clinical case is described relating to an adult woman with the following risk factors: inadequate diet, overweight, sedentary and a family history of heart disease and diabetes. Home visits were made in order to perform an investigation of the socio-demographic characteristics, a baseline survey of eating behavior and physical activity, training in self-monitoring and a final interview. The results point to behavioral changes regarding the implementation and broadening of healthy eating habits and regular physical activity. The discussion of the case includes aspects that indicate the acquisition of preventive behaviors, relating them to the literature used.
\end{abstract}

Uniterms: Diabetes. Reduction of risk factors. Training in self-monitoring.

O diabetes é uma doença crônica que se encontra em crescente epidemia, e muitos portadores dessa patologia desconhecem o diagnóstico, por ela ser, muitas vezes, assintomática (Organização Mundial de Saúde, 2003). Estima-se que atualmente haja no Brasil em torno de onze milhões e meio de portadores de

\section{$\boldsymbol{\nabla} \mathbf{\nabla} \boldsymbol{\nabla}$}

1 Artigo elaborado a partir da dissertação de M.S. CASSEB, intitulada "Prevenção em diabetes: efeitos do treino de automonitoração na redução de fatores de risco". Universidade Federal do Pará, 2005. Apoio: Conselho Nacional de Desenvolvimento Científico e Tecnológico.

2 Universidade da Amazônia, Curso de Psicologia. Av. Alcindo Cacela, 287, Umarizal, 66060-902, Belém, PA, Brasil. Correspondência para/Correspondence to: M.S. CASSEB. E-mails: <casseb4@gmail.com>; <casseb4@yahoo.com.br>.

3 Universidade Federal do Pará, Instituto de Filosofia e Ciências Humanas. Belém, PA, Brasil. 
diabetes, sendo 80\% do tipo 2 (Sociedade Brasileira de Diabetes, 2006). É urgente a aquisição de um estilo de vida saudável por indivíduos que apresentam fatores de risco para o desenvolvimento dessa doença (Absetz et al., 2007; Organização Pan-Americana de Saúde, 2003; World Health Organization - WHO, 2003). Muitos estudos têm obtido resultados positivos com programas comportamentais para auxiliar os indivíduos a reduzir os fatores de risco à saúde, diminuindo, assim, a sobrecarga do tratamento para o diabetes (Absetz et al., 2007; Diabetes Prevention Program, 2002; Glasgow et al., 2001; Swinburn, Metcalf \& Ley, 2001; Wing et al., 2001).

Uma revisão sistemática, realizada por Sartorelli, Franco e Cardoso (2006), aponta a eficácia de intervenções com ênfase na alimentação saudável e prática regular de atividades físicas para prevenir o diabetes tipo 2 e reduzir custos em saúde pública. Em outra revisão sistemática, Yamaoka e Tango (2005) apontaram a eficácia da mudança no estilo de vida para prevenir o diabetes tipo 2 em indivíduos com alto risco, com redução de glicose plasmática duas horas após uma carga de $75 \mathrm{~g}$ de glicose oral e redução da incidência de diabetes em aproximadamente 50\%, se comparado ao grupo-controle nos estudos analisados.

As revisões sistemáticas realizadas por Sartorelli et al. (2006) e por Yamaoka e Tango (2005) compartilham a premissa de que a aquisição de um estilo de vida saudável contribui para a redução da incidência de diabetes. Entretanto, é importante ressaltar que o estilo de vida se caracteriza por padrões comportamentais multideterminados e muitas vezes difíceis de serem modificados. Para minimizar esse problema, propostas de intervenção devem focar mudanças ambientais e comportamentais, considerando a cultura, a realidade e a participação do indivíduo nas decisões (Marrero \& Ackermann, 2007; Ulen, Huizinga, Beech \& Elasy, 2008; $\mathrm{WHO}, 2003)$.

Pesquisadores orientados pela análise aplicada do comportamento têm realizado estudos na área da saúde, priorizando a análise funcional de variáveis ambientais e comportamentais para controle do diabetes (Brandão, 2003; Casseb, Malcher-Bispo \& Ferreira, 2008; Ferreira, 2001; Ferreira \& Casseb, 2002). Vários modelos teóricos da análise aplicada do comportamento utilizam a análise funcional como proposta de intervenção (Ma- proposto por Israel Goldiamond, que consiste na construção e ampliação de comportamentos a partir do repertório que o indivíduo tem (Ferreira, 2001; Goldiamond, 1974).

De acordo com o modelo construcional, repertórios comportamentais são construídos por meio de aproximações sucessivas e reforçamento diferencial (Modelagem), tendo como ponto de partida os comportamentos adequados que o paciente já apresenta. Esse processo de modelagem pode ser auxiliado pelo uso de registros de automonitoração, que treinam o indivíduo para analisar as contingências que controlam seu comportamento, possibilitando a auto-observação de ganhos obtidos (Bohm \& Gimenes, 2008; Ferreira 2001).

Automonitoração refere-se a observações que o indivíduo faz do próprio comportamento por meio do seu registro e de fatores que o influenciam, permitindo a condução de análises funcionais para delimitação dos objetivos de intervenção, planejamento e avaliação dos resultados (Bohm \& Gimenes, 2008; Tobin, Reynolds, Holroyd \& Creer, 1986). Na área de saúde, o treino em automonitoração tem se mostrado eficaz em diversos estudos para modificar hábitos e estilos de vida prejudiciais à saúde (Brandão, 2003; Duchesne et al., 2007; Ferreira \& Casseb, 2002; Marrero \& Ackermann, 2007; Ulen et al., 2008).

Diante dessa perspectiva, o objetivo deste estudo foi verificar se o treino em automonitoração mostra-se eficaz na instalação e na manutenção de comportamentos preventivos correspondentes à dieta e à prática de atividade física e na redução de fatores de risco para diabetes tipo 2, por meio da apresentação de um caso clínico.

\section{Método}

\section{Participante}

Participou deste estudo uma mulher de 41 anos de idade, com domínio de leitura e escrita, e apresentando fatores de risco para o desenvolvimento de diabetes. Considerando-se os aspectos éticos da pesquisa, a participante foi solicitada a assinar o termo de consentimento livre e esclarecido; para resguardar sua identidade, foi-Ihe dado o nome fictício de Laura (Projeto 
aprovado pelo Comitê de Ética em Pesquisas em Seres Humanos, Protocolo no 098/2004-CEP/NMT).

\section{Instrumentos}

1) Roteiro de entrevista 1: roteiro semiestruturado para identificar dados sociodemográficos e levantar os conhecimentos da participante acerca do diabetes.

2) Recordatório de 24 horas: roteiro para a participante relatar as atividades realizadas no dia anterior à entrevista quanto à alimentação e à atividade física.

3) Protocolo nutricional do serviço de nutrição: protocolo impresso fornecido pelo serviço de nutrição, contendo regras nutricionais individualizadas para a participante, para adequação da dieta.

4) Folder sobre diabetes do serviço de nutrição: folder explicativo com informações sobre definição, prevenção e tratamento do diabetes, fornecido à participante durante a consulta com a nutricionista.

5) Protocolos de automonitoração: elaborados para que a participante registrasse por escrito seu comportamento alimentar e de atividade física. Foram utilizados três tipos de protocolos: (1) registro diário de alimentação, com colunas para o registro da alimentação quanto à qualidade, quantidade e horários das refeições; (2) registro de planejamento diário de alimentação, com colunas para o registro das refeições a serem realizadas no dia seguinte à entrevista, incluindo qualidade, quantidade e horário das refeições, seguidas de uma coluna para o registro dos comportamentos emitidos; e (3) registro semanal de atividade física, com colunas correspondentes a cada dia da semana, nas quais a participante deveria marcar com um X o dia em que realizasse a atividade física orientada pelo profissional de saúde.

6) Roteiro de entrevista 2: roteiro elaborado com o objetivo de auxiliar a participante a fazer a análise da adesão à dieta, comparando-a com as regras do protocolo nutricional. Tal comparação permitia o cálculo do Índice de Adesão à Dieta (IAD), uma vez que, para cada refeição correspondente às regras, era atribuído um ponto; caso contrário, não era atribuído o ponto. Ao final, obtinha-se a soma do número de refeições corretas, a qual era multiplicada por 100; em seguida, esse valor era dividido pelo número total de refeições $(n=6)$ que deveriam ter sido realizadas ao longo do dia (desjejum, lanche 1, almoço, lanche 2, jantar e ceia), sendo obtido o IAD na forma de percentagem. Esse cálculo considerou o fracionamento, a qualidade e a quantidade dos alimentos, separadamente e em conjunto.

7) Escala de avaliação da adesão à dieta e à atividade física: elaborada para auxiliar a participante na autoavaliação da adesão, considerando-a como boa, regular ou baixa.

8) Quadro cumulativo da escala de avaliação da adesão: elaborado para demonstrar à participante, na forma de feedback positivo, as avaliações acumuladas e seu comportamento de adesão à dieta e à atividade física durante a pesquisa.

9) Roteiro de entrevista 3: roteiro semiestruturado para avaliar a participação de Laura no estudo, obtendo uma descrição sobre os benefícios e custos da adesão às orientações sobre prevenção em diabetes.

\section{Procedimentos}

Após a seleção da participante, a coleta de dados foi realizada em dois meses e meio, por meio de 13 entrevistas gravadas em áudio, com um intervalo médio de dois dias entre as visitas domiciliares.

O estudo obedeceu às seguintes etapas: (i) entrevista inicial para levantamento de dados sociodemográficos e conhecimentos sobre diabetes e comportamentos preventivos, utilizando-se o roteiro de entrevista 1; (ii) investigação da Linha de Base 1 do comportamento alimentar e da atividade física, utilizando-se o recordatório de 24 horas antecedentes à entrevista; (iii) consulta da participante com o Serviço de Nutrição de um Hospital Universitário, com a obtenção do protocolo nutricional, folder sobre diabetes, e instruções sobre a execução de atividade física regular, por três dias na semana, durante 15 a 30 minutos; (iv) investigação da Linha de Base 2 do comportamento alimentar e de práticas de atividade física, efetuada em ambiente domiciliar uma semana após a participante ser consultada com o Serviço de Nutrição de um hospital universitário, utilizando-se o recordatório de 24 horas; (v) treino em automonitoração do comportamento alimentar e da atividade física, realizado em 11 visitas domiciliares em dias alternados, utilizando-se os protocolos de 
registros de automonitoração (iniciando-se com o Registro diário de alimentação, seguido do Registro de planejamento diário de alimentação), o roteiro de entrevista 2, a escala de avaliação da adesão à dieta e à atividade física, e o quadro cumulativo da escala de avaliação da adesão (a participante avaliava se sua adesão estava boa, regular ou baixa e registrava, ao final de cada semana, na escala de avaliação da adesão à dieta e à atividade física; ao final da intervenção, as avaliações foram registradas pela pesquisadora no Quadro cumulativo de avaliação da adesão, que foi apresentado para fornecer feedback à participante); (vi) Nova consulta com o serviço de nutrição; e (vii) Entrevista Final, com a aplicação do roteiro de entrevista 3.

\section{Resultados}

\section{Descrição do caso clínico}

Laura tinha ensino fundamental completo, estava desempregada e morava com uma irmã, a mãe e 11 crianças. A renda familiar era de um salário-mínimo mensal, obtido por meio da aposentadoria da mãe.

Laura apresentava fatores de risco para o desenvolvimento do diabetes, como histórico familiar de diabetes e cardiopatia, diagnóstico de sobrepeso (índice de massa corporal=26), ausência de atividade física regular e hábitos alimentares inadequados quanto ao

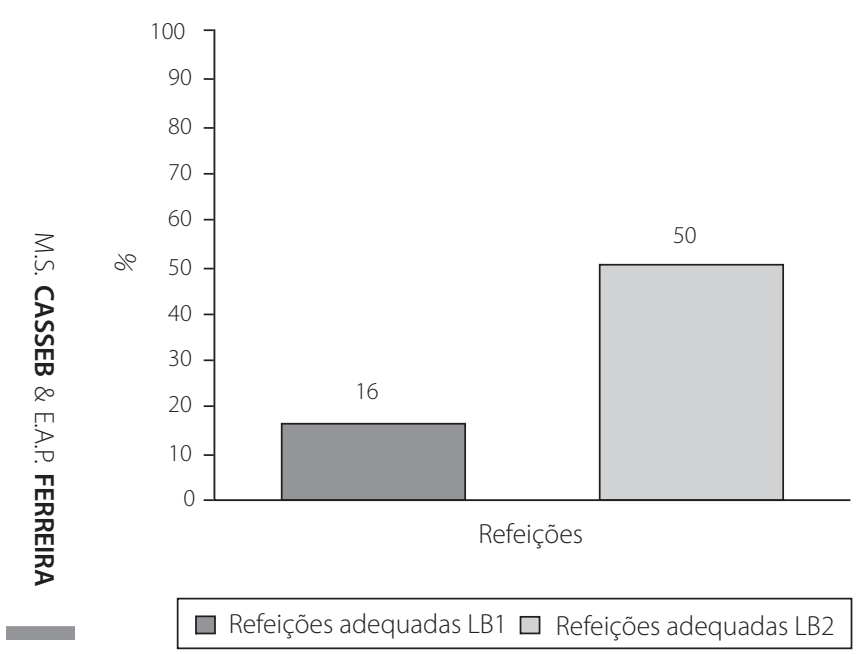

fracionamento, à qualidade e à quantidade de alimentos ingeridos. Além disso, a participante tinha conhecimentos insuficientes sobre diabetes e sobre como prevenir-se da doença, o que pode ter favorecido o desenvolvimento de fatores de risco.

\section{Repertório inicial}

\section{Linha de Base do comportamento alimentar e da atividade física}

Durante o levantamento da primeira Linha de Base (LB) do comportamento alimentar (LB1), Laura apresentou 16\% de índice de adesão à dieta, o que equivale a somente uma refeição adequada durante um período de 24 horas. Quanto aos resultados da utilização da segunda linha de base do comportamento alimentar (LB2), obtida uma semana após Laura ter se consultado com a nutricionista, verifica-se que, comparada à LB1, houve melhora no índice de adesão à dieta após a consulta com a nutricionista (Figura 1). Quanto aos aspectos da alimentação, observa-se que, em LB2, houve melhora no fracionamento se comparado à qualidade e à quantidade das refeições.

No que se refere à atividade física, durante a entrevista inicial, Laura considerou a caminhada como a atividade mais fácil de executar, planejando realizá-la durante uma hora ao dia. Entretanto, durante a LB1 Laura

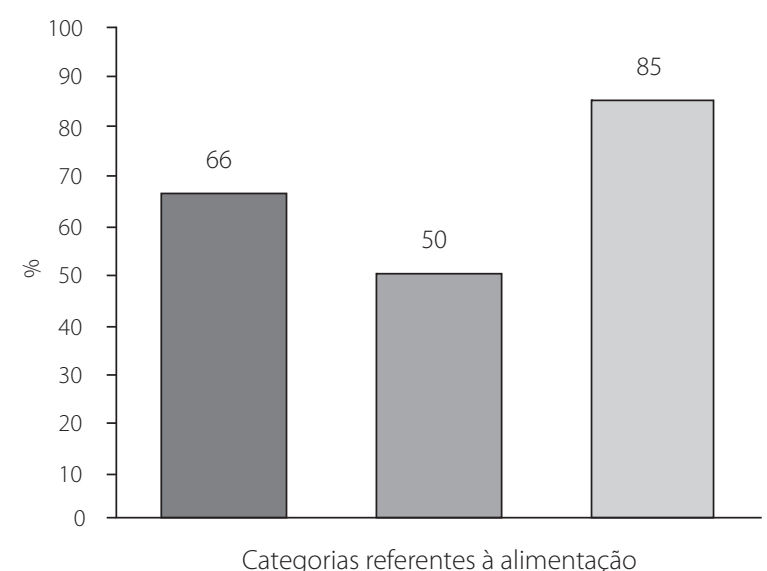

Quantidade Qualidade Fracionamento

138 Figura 1. Índices de adesão à dieta obtidos por Laura durante LB1 e LB2. Belém (PA), 2004. 
não realizou nenhuma caminhada, mantendo esse padrão durante a LB2, após a consulta com a nutricionista, sob alegação de dificuldades para incluir tal atividade em sua rotina.

\section{Intervenção}

O treino em automonitoração da alimentação que exige um acompanhamento mais frequente, foi realizado por meio de visitas domiciliares, a fim de se evitar custo para a participante. As visitas também permitiram à pesquisadora observar melhor a realidade e a rotina da participante, a fim de auxiliá-la a estabelecer as mudanças necessárias à adesão.

No que se refere à adesão à dieta, incluindo-se todos os aspectos da alimentação (fracionamento, qualidade e quantidade), verifica-se que Laura apresentou índice igual ou acima de 50\% nas refeições executadas (Figura 2-A). Comparando-se esses dados com os 16\% de adesão obtidos durante as LB1 e LB2 (Figura 1), verifica-se uma diferença mínima de 34\% (R6, R9, R10) e máxima de 67\% (R1, R2, R3, R8, R11) na adesão à dieta.
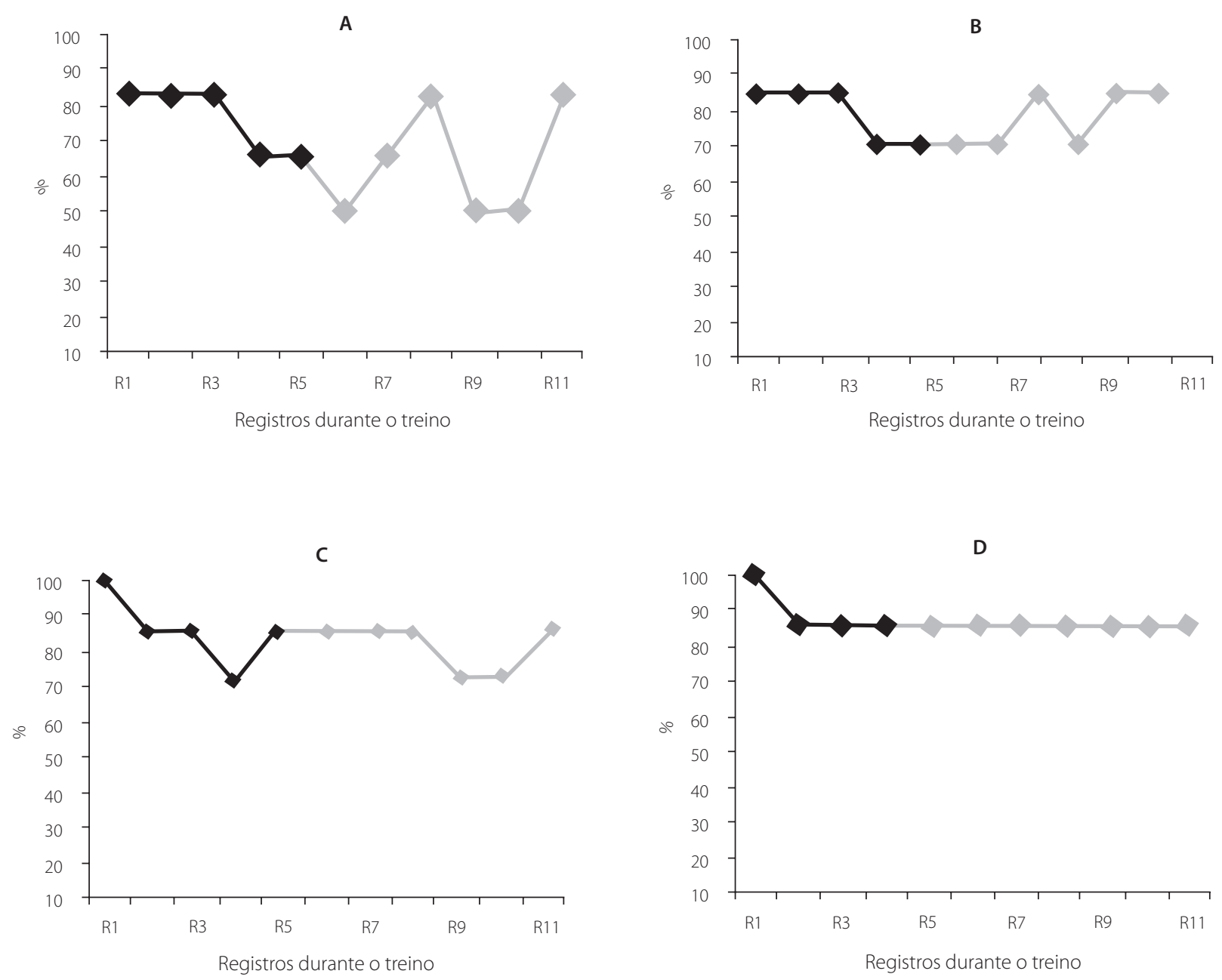

$\longrightarrow$ Registro diário da alimentação

$\longrightarrow$ Registro de planejamento diário da alimentação

Figura 2. Índice de adesão à dieta, quantidade, qualidade e fracionamento da alimentação durante o treino de automonitoração. A) Índice de adesão à dieta; B) Índice de adesão à quantidade de alimentos nas refeições; C) Índice de adesão à qualidade de alimentos nas refeições; D) Índice de adesão ao fracionamento das refeições. Belém (PA), 2004. 
Observa-se que houve maior estabilidade na adesão ao fracionamento das refeições, sugerindo que Laura teve maior facilidade para estabelecer mudanças nesse aspecto apesar de ter mantido um IAD igual ou acima de 50\% para quantidade e qualidade das refeições. Os dados apontam que o treino de automonitoração auxiliou na adesão à dieta pela participante (Figuras 2B, (eD).

\section{Treino em automonitoração da atividade física}

No início desse treino, Laura registrou a inclusão da caminhada em suas atividades de rotina, como ir ao hospital auxiliar a mãe que havia sofrido um acidente ou levar seus filhos à escola, apesar de não ter o hábito de sair de casa com frequência, conforme relatado na entrevista inicial.

No retorno à consulta com a nutricionista, Laura recebeu novas orientações sobre como fazer a caminhada e iniciou essa atividade de forma mais regular, registrando uma frequência de 3 a 4 vezes por semana, durante trinta minutos. Durante o acompanhamento, Laura referiu que caminhou três vezes durante a semana por trinta minutos e que não se sentiu desconfortável fisicamente.

\section{Encerramento}

No encerramento da pesquisa, Laura considerou a alimentação como a parte do treino mais fácil de realizar, ao compará-la à atividade física. Quanto aos ganhos obtidos com a intervenção, Laura relatou a orientação recebida sobre prevenção do diabetes e melhora nos cuidados com a alimentação. Sobre a qualidade de vida, Laura avaliou que houve ganhos, mas que ainda precisava incluir lazer em sua rotina, apesar de já estar consciente dessa necessidade.

\section{Discussão}

Os resultados referem-se à análise da adesão da participante a comportamentos preventivos referentes à dieta e à atividade física. No que se refere à dieta, os resultados do treino sugerem que o uso dos registros de automonitoração auxiliou a participante na instalação de comportamentos de autoobservação e de relatar com acurácia o comportamento alimentar, o que confirma os resultados de estudos anteriores que utilizaram procedimentos de automonitoração para promover a adesão ao tratamento (Brandão, 2003; Casseb, Malcher-Bispo \& Ferreira, 2008; Ferreira, 2001; Ferreira \& Casseb, 2002) e demonstra que o treino também auxilia a construção e a ampliação de comportamentos preventivos.

Dentre as variáveis que facilitaram a adesão à dieta pela participante, destacam-se a avaliação que ela fazia de sua própria adesão, as regras fornecidas pela nutricionista, o fornecimento de feedback positivo contingente ao relato da adesão e o uso dos registros de automonitoração, como sugere a literatura (Absetz et al., 2007; Bohm \& Gimenes, 2008; Ferreira \& Casseb, 2002).

Como variáveis que mais dificultaram a adesão à dieta foram identificadas a restrição financeira para acesso aos alimentos indicados, apoio social restrito, dificuldade em compreender e analisar a relação custo-benefício de aderir às orientações, falta de habilidades para adequar a rotina ao seguimento das instruções, o que confirma estudos anteriores sobre adesão à dieta (Casseb, Malcher-Bispo \& Ferreira, 2008; Sociedade Brasileira de Diabetes, 2002; Ferreira, 2001; Ferreira \& Casseb, 2002; WHO, 2003b, Wing et al., 2001).

Quanto à atividade física, o treino de automonitoração permitiu que Laura refinasse a auto-observação de seu comportamento, avaliando os benefícios da atividade como superiores aos custos de executá-la. Desse modo, os resultados confirmam os benefícios do uso de registros de automonitoração na mudança de comportamentos (Bhom \& Gimenes, 2008; Casseb et al., 2008).

Como variáveis que facilitaram a adesão à atividade física, destacam-se o uso dos registros de automonitoração, a análise funcional constante da relação custo-benefício para fazer exercícios, a discriminação dos ganhos obtidos, a autoavaliação da adesão, a liberação de feedback e reforçamento positivo pela pesquisadora e a instalação gradual de atividade física na rotina. Tais variáveis auxiliaram na redução de eventos aversivos 
diretamente relacionados com a execução dos exercícios, confirmando a literatura (Diabetes Prevention Program, 2002; Ulen et al., 2008). Uma das variáveis que dificultaram a adesão de Laura à caminhada foi seu deficit em habilidades para identificar as contingências que dificultavam a organização de sua rotina e a falta de apoio social.

\section{Considerações Finais}

Variáveis culturais, sociodemográficas, biológicas e ambientais exercem influência sobre comportamentos de adesão, podendo facilitar ou dificultar a construção e a ampliação de comportamentos preventivos de doenças crônicas, tais como o diabetes. Entretanto, o uso de registros de automonitoração, aliado ao apoio social, feedback, reforçamento positivo, recursos didáticos (registros, diários, textos informativos) e instruções sobre análise de contingências podem contribuir para a redução das dificuldades e consequente aumento da adesão a comportamentos de prevenção em saúde. É necessário também o acompanhamento sistemático, que pode ser feito por meio de visitas domiciliares com intervenções que priorizem as peculiaridades e a realidade de cada indivíduo, visto que a adesão às regras exige uma mudança comportamental e ambiental muitas vezes difícil de ser estabelecida.

O treino em automonitoração foi utilizado neste estudo considerando-se o repertório que a participante apresentava antes do treino, a relação custo-benefício para a emissão de uma dada resposta, o contexto em que o comportamento ocorria e o estabelecimento gradativo de mudanças. Considera-se que essas variáveis são de grande importância ao se implementar a educação para prevenção do diabetes, tendo em vista os custos que um tratamento pode impor ao paciente.

Por se tratar de um caso clínico, as dificuldades deste estudo estão na generalização dos resultados, tendo em vista o tempo de aplicação do procedimento. Sugere-se a replicação com maior número de pessoas em risco para desenvolver diabetes e também com profissionais de saúde para verificar os efeitos sobre a construção e a ampliação de comportamentos adequados na atenção ao paciente.

\section{Referências}

Absetz, P., Valve, R., Oldenburg, B., Heinonen, H., Nissinen, A., Fogelholm, M., et al., (2007). Type 2 diabetes prevention in the "Real World": one-year results of the GOAL implementation trial. Diabetes Care, 30 (10), 2465-2470.

Bohm, C. H., \& Gimenes, L. S. (2008). Automonitoramento como técnica terapêutica e de avaliação comportamental. Revista de Psicologia, 1 (1), 88-100.

Brandão, W. L. O. (2003). Adesão ao tratamento por pacientes portadores de diabetes tipo 1 e tipo 2: efeitos do treino de discriminação de dicas internas e externas. Dissertação de mestrado não-publicada, Universidade Federal do Pará, Belém.

Casseb, M. S., Malcher-Bispo, M. S., \& Ferreira, E. A. P. (2008). Automonitoração e seguimento de regras nutricionais em diabetes: dois studos de caso. Interação em Psicologia, $12(2), 223-233$.

Diabetes Prevention Program. (2002). Description of lifestyle intervention. Diabetes Care, 25 (12), 2165-2171.

Duchesne, M., Appolinário, J. C., Rangé, B. P., Freitas, S., Papelbaum, M., \& Coutinho, W. (2007). Evidências sobre a terapia cognitivo-comportamental no tratamento de obesos com transtorno da compulsão alimentar periódica. Revista de Psiquiatria do Rio Grande do Sul, 29(1), 80-92.

Ferreira, E. A. P. (2001). Adesão ao tratamento em portadores de diabetes: efeitos de um treino em análise de contingências sobre comportamentos de autocuidado. Tese de doutorado não-publicada, Instituto de Psicologia, Universidade de Brasília.

Ferreira, E. A. P., \& Casseb, M. S. (2002). Efeitos do uso de registros de automonitoração no seguimento de regras nutricionais por uma portadora de diabetes Tipo 2 e obesidade classe I. Diabetes Clínica (Rio de Janeiro), 6 (6), 452-459.

Glasgow, R. E., Hiss, R. G., Anderson, R. M., Friedman, N. M., Hayward, R. A., Marrero, D. G., et al. (2001). Report of the health care delivery work group: behavioral research related to the establishment of a chronic disease model for diabetes care. Diabetes Care, 24 (1), 124-130.

Goldiamond, I. (1974). Toward a construcional approach to social problems: ethical and constitutional issues raised by applied behavior analysis. Behaviorism, 2 (1), 1-84.

Marrero, D. G., \& Ackermann, R. T. (2007). Providing longterm support for lifestyle changes: A key to success in diabetes prevention. Diabetes Spectrum, 20 (4), 205-209.

Matos, M. A. (1999). Análise funcional do comportamento. Estudos de Psicologia (Campinas), 16 (3), 8-18.

Organização Mundial de Saúde. (2003). Cuidados inovadores para condições crônicas: componentes estruturais de ação: relatório mundial. Brasília: OMS. Recuperado em fevereiro 15, 2004, disponível em <http://www.opas.org.br/ publicmo.cfm?codigo $=62>$.

Organização Pan-Americana de Saúde. (2003). Doenças crônico-degenerativas eobesidade: estratégia mundial sobre alimentação saudável, atividadefísica esaúde. Brasilia: OPAS. 
Recuperado em janeiro 11, 2011, disponível em <http:// www.opas.org.br/publicmo.cfm?codigo $=66>$.

Sartorelli, D. S., Franco, L. J., \& Cardoso, M. A. (2006). Intervenção nutricional e prevenção primária do diabetes mellitus tipo 2: uma revisão sistemática. Cadernos de Saúde Pública, 22 (1), 7-18.

Sociedade Brasileira de Diabetes. (2006). Atualização brasileira sobre diabetes. Diretrizes da Sociedade Brasileira de Diabetes. Recuperado em maio 11, 2008, disponível em <http://www.diabetes.org.br/educacao/docs/atualizacao diabetes2006.pdf>.

Swinburn, B. A., Metcalf, P. A, \& Ley, S. J. (2001). Long-term (5-Year) effects of a reduced- fat diet intervention in individuals with glucose intolerance. Diabetes Care, 24 (4), 619-624

Tobin, D. L., Reynolds, R. V. C., Holroyd, K. A., \& Creer, T. L. (1986). Self-management and social learning theory. In K. A. Holroyd \& T. L. Creer (Eds.), Self-management of chronic disease: handbook of clinical interventions and research (pp.29-55). Orlando, FL: Academic Press.
Ulen, C. G., Huizinga, M.M., Beech, B., \& Elasy, T. A. (2008). Weight regain prevention. Clinical Diabetes, 26 (3), 100-113.

Wing, R. R., Goldstein, M. G., Acton, K. J., Birch, L. L., Jakicic, J. M., Sallis Jr., J. F., et al. (2001). Behavioral science research in diabetes: lifestyle changes related to obesity, eating behavior, and physical activity. Diabetes Care, 24 (1), 117-123.

World Health Organization. (2003). Diet, nutrition and the prevention of chronic diseases: report or a joint WHO/FAO expert consultation. Geneva, Retrieved August 12, 2003, from <http://www.who.int/publications/en/>.

Yamaoka, K., \& Tango, T. (2005). Efficacy of life stile education to prevent type 2 diabetes: a meta-analysis of randomized controlled trials. Diabetes Care, 28 (11), 2780-2786.

Recebido em: 9/12/2008

Versão final reapresentada em: 16/9/2011

Aprovado em: 7/10/2011 